San Jose State University

SJSU ScholarWorks

Master's Theses

Master's Theses and Graduate Research

Spring 2012

\title{
The Investigation of the UNC-6/Netrin and UNC-40/DCC-mediated Synaptic Partner Recognition Pathway
}

Kelli Benedetti

San Jose State University

Follow this and additional works at: https://scholarworks.sjsu.edu/etd_theses

\section{Recommended Citation}

Benedetti, Kelli, "The Investigation of the UNC-6/Netrin and UNC-40/DCC-mediated Synaptic Partner Recognition Pathway" (2012). Master's Theses. 4120.

DOI: https://doi.org/10.31979/etd.9ekq-tgqz

https://scholarworks.sjsu.edu/etd_theses/4120

This Thesis is brought to you for free and open access by the Master's Theses and Graduate Research at SJSU ScholarWorks. It has been accepted for inclusion in Master's Theses by an authorized administrator of SJSU ScholarWorks. For more information, please contact scholarworks@sjsu.edu. 
THE INVESTIGATION OF THE UNC-6/NETRIN AND UNC-40/DCC-MEDIATED SYNAPTIC PARTNER RECOGNITION PATHWAY

\author{
A Thesis \\ Presented to \\ The Faculty of the Department of Biological Sciences \\ San José State University \\ In Partial Fulfillment \\ of the Requirements for the Degree \\ Master of Science
}

by

Kelli Benedetti

May 2012 
(C) 2012

Kelli Benedetti

ALL RIGHTS RESERVED 
The Designated Thesis Committee Approves the Thesis Titled

THE INVESTIGATION OF THE UNC-6/NETRIN AND UNC-40/DCC-MEDIATED SYNAPTIC PARTNER RECOGNITION PATHWAY
by
Kelli Benedetti
APPROVED FOR THE DEPARTMENT OF BIOLOGICAL SCIENCES
SAN JOSÉ STATE UNIVERSITY
May 2012
Dr. Miri VanHoven Department of Biological Sciences
Dr. Robert Fowler Department of Biological Sciences
Dr. Rachael French Department of Biological Sciences 


\section{ABSTRACT \\ THE INVESTIGATION OF THE UNC-6/NETRIN AND UNC-40/DCC-MEDIATED SYNAPTIC PARTNER RECOGNITION PATHWAY}

by Kelli Benedetti

Organisms receive, process, and respond to environmental cues via intricate neural circuits that form during development. Proper development of neuronal circuits is therefore critical to brain function. To form these circuits, a neuron must first extend its axons to the appropriate target. After reaching this target region, it must recognize its correct synaptic partners from multiple incorrect partners and form appropriate synapses. It is well understood how axons reach their target regions, but little is known about how neurons select the correct partners once there, a process called synaptic partner recognition (SPR). Our work aims to elucidate the genetic programs that specify correct SPR in sensory circuits in the nematode Caenorhabditis elegans. We used the fluorescent trans-synaptic marker, Neuroligin 1-mediated GFP Reconstitution Across Synaptic Partners (NLG-1 GRASP). Using this marker, we discovered a role for an UNC-6/Netrin and UNC-40/DCC-mediated axon guidance pathway gene that functions in SPR. Interestingly, synaptic component localization in mutants of this gene is normal, indicating that the reduced SPR is not the result of a secondary defect in cell polarity or protein trafficking. Finally, we found that this gene functions in the UNC-6/Netrin and UNC-40/DCC SPR pathway previously described by our laboratory. Most remarkably, we found that this new gene's function in the SPR pathway is molecularly distinct from its previously characterized signaling pathways. The characterization of the novel role of this gene may give insights into the mechanism of sensory circuit formation. 


\section{ACKNOWLEDGEMENTS}

I would like to first thank my undergraduate and graduate adviser, Professor Miri VanHoven. She helped excite my interest in genetics and molecular and cellular neuroscience. Without her, I definitely would not have pursued graduate education. I greatly cherish all of her valuable help, guidance, and mentoring over the past three years. I would also like to thank the rest of my thesis committee, Professor Robert Fowler and Professor Rachael French for all of their help and guidance. I would also like to thank Professor Rachael French for being a great professor, and Professor Robert Fowler for being great to teach with. Lastly, I would like to thank all previous, past, and present members of the VanHoven lab for all of their help and support over the past few years.

All of the experiments in this thesis will be submitted for publication in a peerreviewed journal later this year. This work was funded by the US National Institutes of Health (MBRS SC3 \#GM089595 to MKV), MARC \#5T34GM008253 undergraduate fellowship to KB), and the San José State University Research Foundation (Davison Fellowship to $\mathrm{KB}$ ). The funders had no role in study design, data collection and analysis, or preparation of this thesis. 


\section{TABLE OF CONTENTS}

LIST OF FIGURES..................................................viii

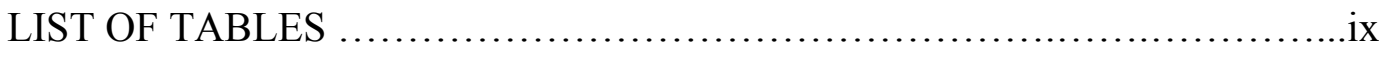

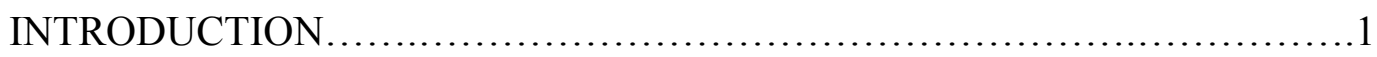

EXPERIMENTAL PROCEDURES......................................9

Strains and genetics.........................................

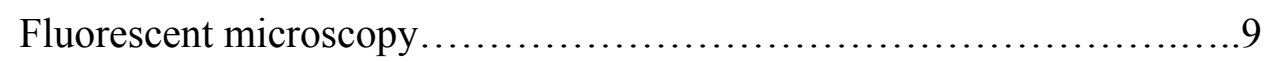

Phenotypic quantification...................................... 10

Statistical analysis............................................ 10

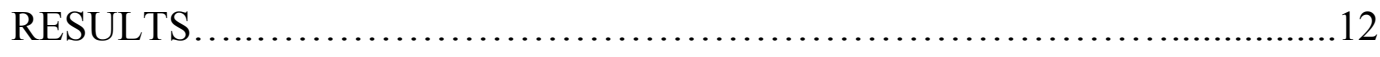

clr-1 mutants have defective Synaptic Partner Recognition..............12

CLR-1 functions in the UNC-6/Netrin and UNC-40/DCC-mediated SPR pathway...............................................

Synaptic components are properly localized in $c l r-1$ mutants............16

Molecules that function with CLR-1 or with RPTPs in other developmental pathways do not mediate SPR........................19

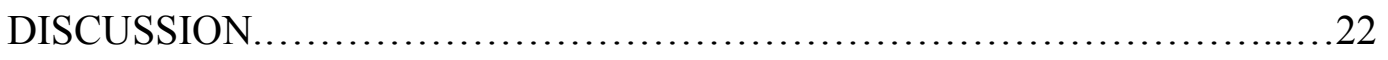

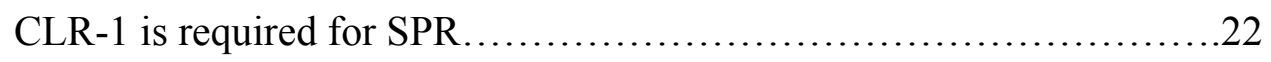


CLR-1 functions in the UNC-6/Netrin and UNC-40/DCC-mediated

SPR pathway................................................24

Incorrect SPR in clr-1 mutants is the result of a primary recognition defect and not due to incorrect synaptic component localization...........28

The CLR-1 SPR pathway is distinct from previously described RPTP

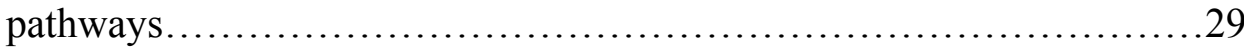

Implications of SPR in understanding proper brain function...........29

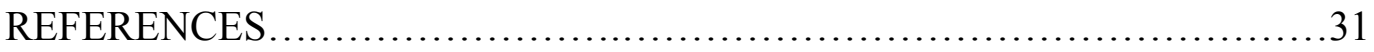




\section{LIST OF FIGURES}

Figure 1. $c l r-1$ mutants display defective SPR and CLR-1 functions in the

UNC-6/Netrin and UNC-40/DCC-mediated SPR pathway .........................15

Figure 2. Vesicle trafficking, active zone, and postsynaptic components localize to the correct subcellular compartment in $c l r-1$ mutants................................18

Figure 3. RPTP pathway mutants exhibit no defects in SPR ........................20

Figure 4. UNC-6/Netrin, UNC-40/DCC, and CLR-1 mediate SPR between presynaptic PHB sensory neurons and postsynaptic AVA interneurons.................27 


\section{LIST OF TABLES}

Table 1. Summary of statistical analysis.......................................24 


\section{INTRODUCTION}

Neurons relay information to each other through specialized structures called

synapses. Chemical synapses are junctions formed between presynaptic and postsynaptic neurons that allow information to be transferred directionally. Information is transmitted in the form of neurotransmitters that are secreted by the presynaptic neuron. These neurotransmitters move across the presynaptic cleft and subsequently bind to receptors located at postsynaptic sites, eliciting the proper response from the postsynaptic cells.

Neurons are connected via synapses into functional units called circuits that mediate perception, thought and behavior. Much like an electrical circuit, neurons are connected to each other to transmit signals necessary for proper function of the nervous system. These circuits are formed during development, creating simple or intricate connections between neurons. An example is the mammalian olfactory circuit in which sensory neurons, called olfactory receptor neurons, sense specific odorants and form synapses with appropriate interneurons in a region of the brain called the olfactory bulb. These signals are eventually carried to the olfactory cortex via interneurons called mitral cells (Brennan and Keverne, 1997). Failure to form a neural circuit correctly results in a failure of neural function. For instance, a failure of the olfactory sensory neurons to form synapses with appropriate interneurons would result in failure to sense the corresponding odorant. However, widespread defects in neural circuit formation could result in more serious functional deficits and have been linked with neurological disorders, such as 
autism and schizophrenia. Therefore, it is critical to understand how neural circuit formation is accomplished during development.

To form a neural circuit, neurons must identify their correct synaptic partners, a process called synaptic partner recognition (SPR). Early in development, neurons extend long cellular extensions called neurites into target regions containing the correct synaptic partners. However, these regions usually contain many other neurites. Electron micrograph reconstruction studies in invertebrates and vertebrates have shown that neurons can distinguish their appropriate synaptic partners, despite contacting many other cells (Shen and Scheiffele, 2010). Thus, it is critical that neurites recognize and form synapses with only the correct partners. This reliable process allows for proper neural circuit formation during development. However, the molecular mechanisms underlying SPR are poorly understood.

To understand SPR, we study the genetic model organism Caenorhabditis elegans. C. elegans is a microscopic, free-living nematode that is an excellent model for studying genetics. Not only is the entire genome sequenced, but there are various molecular tools, including cell-specific promoters for genes and cell-specific RNAi. Additionally, C. elegans is an excellent model in which to address questions about neural development. C. elegans has only 302 neurons, connected by 7000 synapses. In comparison, the human brain has approximately one hundred billion neurons connected by trillions of synapses. Thus, this model allows for feasible studies of specific neuronal interactions. Another important feature of C. elegans is that the morphology of the synapses and the components localized to the synapses are largely conserved in 
vertebrates, making it an ideal model for neuroscience research (Shen et al., 2004; Zhen and Jin, 1999). However, arguably the best feature of C. elegans for use in neuroscience research is that the entire synaptic connectivity has been mapped through decades of electron micrograph (EM) reconstruction (White et al., 1976; White et al., 1986). This has allowed us to study the genetics of SPR between specific cells that have been previously shown to form synapses with each other.

To study the molecular signals that mediate SPR, we have employed a transgenic fluorescent synaptic marker called Neuroligin-1 (NLG-1) Green Fluorescent Protein (GFP) Reconstitution Across Synaptic Partners, or NLG-1 GRASP. Previously, the only way to study SPR was through electron microscopy. Therefore these studies could only be performed on sections of fixed specimens. Additionally, analysis of a single animal with electron microscopy is time-consuming, requiring months to years to analyze a single animal. To create a much more rapid assay that can be performed in live animals, we developed NLG-1 GRASP (Feinberg et al., 2008). This marker is based on split GFP. The molecule is split into two fragments: one containing beta sheets 1-10 of GFP and a second containing only beta sheet 11 of GFP. The split GFP fragments are tethered to the extracellular domain of NLG-1, a transmembrane protein that localizes to both pre- and postsynaptic sites in C. elegans. The fragments can be expressed in pre- and postsynaptic neurons of interest using cell-specific promoters. When synapses between specific neurons are formed, the GFP fragments reconstitute and fluoresce. However, if the correct partners fail to form a synapse, fluorescence is lost (Feinberg et al., 2008). Importantly, our previous studies indicate that this marker does not generate ectopic 
synapses (Park et al., 2011). Thus, NLG-1 GRASP allows instant identification of correct SPR in specific neural circuits in live animals.

In this study, we test the molecules that mediate SPR between PHB sensory neurons, which are responsible for sensing noxious liquids, and AVA interneurons, which control backward movement of the worm in response to contact with such liquids. This interaction is an excellent model for SPR because the PHB sensory neurons faithfully form synapses with AVA interneurons despite contacting approximately 30 other neurons (Hall and Russell, 1991; White et al., 1986).

There are two types of synapses: terminal synapses, which form between the termini of pre- and postsynaptic neurites, and en passant synapses, which form between parallel pre- and postsynaptic neurites. Vertebrates form both types of synapses, while $C$. elegans primarily forms en passant synapses. In both cases, the correct synaptic partner must be among the neurites that a presynaptic neuron contacts for an appropriate synapse to form. To visualize neurite contact between our neurons of interest, we cell-specifically express a cytosolic red fluorophore called mCherry in pre- and postsynaptic neurons to completely label the neurites. This allows for contact to be assessed. Once proper contact is confirmed, synaptic intensity can be studied.

Previously, we uncovered a novel role for a conserved receptor-ligand pair in SPR using NLG-1 GRASP technology (Park et al., 2011). UNC-6 is the C. elegans homolog of the vertebrate protein Netrin, while UNC-40 is the C. elegans DCC (Deleted in Colorectal Cancer) homolog. UNC-6/Netrin is a diffusible, secreted ligand related to vertebrate Laminin proteins, whereas UNC-40/DCC is a transmembrane receptor that is 
part of the Immunoglobulin Superfamily (IgSF) of proteins. UNC-6/Netrin and UNC40/DCC are a very well-studied receptor-ligand pair, characterized extensively for their earlier roles in neural circuit formation (Moore et al., 2007; Tessier-Lavigne and Goodman, 1996). These roles include cell migration, axon guidance, and targeting of synaptic components. UNC-6/Netrin has an attractive cue for cells expressing UNC40/DCC. Axons expressing UNC-40/DCC grow towards increasing concentrations of UNC-6/Netrin (Dickson, 2002; Serafini et al., 1994). Conversely, axons expressing the UNC-5/UNC5 receptor with or without UNC-40/DCC are repelled by UNC-6/Netrin (Hedgecock et al., 1990).

Our group found severe and highly penetrant defects in SPR in both unc-6/Netrin and $u n c-40 / D C C$ mutant animals, indicating that both genes are required for PHB-toAVA SPR. Further, we found that UNC-6/Netrin and UNC-40/DCC function in a juxtacrine manner, with UNC-40/DCC expressed presynaptically and UNC-6/Netrin expressed postsynaptically, to mediate SPR between PHB and AVA neurons (Park et al. 2011).

Our next goal was to identify additional molecules in the UNC-6/Netrin and UNC-40/DCC pathway that mediate SPR. Several other molecules have been placed in the canonical Netrin/DCC-mediated axon guidance pathway, including co-receptors SDN-1/Syndecan and GPN-1/Glypican (Fox and Zinn, 2005; Johnson et al., 2006), both of the Heparan Sulfate Proteoglycan (HSPG) proteins, upstream cues from MIG-2/Rho, VAB-8/Kinesin, UNC-73/Trio (Levy-Strumpf and Culotti, 2007), CLR-1, and the 
downstream effectors CED-10/Rac1, UNC-34/Enabled (Gitai et al., 2003), UNC115/abLIM (Lundquist et al., 1998), and NCK-1/Nck1 (Li et al., 2002).

We tested 35 candidate molecules for roles in SPR utilizing the NLG-1 GRASP marker in mutant worms. We found that the most highly penetrant and severe SPR defects were found in $c l r-1$, for clear mutant 1 , animals. CLR-1 is a receptor protein tyrosine phosphatase (RPTP), previously studied for its role in fluid regulation in the pseudocoelom (Kokel et al., 1998) and repulsion of UNC-6/Netrin and UNC-40/DCCmediated axon guidance (Chang et al., 2004).

Receptor protein tyrosine phosphatases (RPTPs) have been studied for their important roles in neuronal morphogenesis in both invertebrates and vertebrates. However, unlike the well-studied receptor tyrosine kinases (RTKs), the mechanisms by which these receptors function are poorly understood. Additionally, the molecular pathways have not been thoroughly elucidated. RPTPs have been studied for their in vivo roles in axon outgrowth and guidance, as well as in neuromuscular junction (NMJ) growth (Johnson and Van Vactor, 2003). LAR is a RPTP that has been studied in detail in Drosophila for its various roles in neural development. LAR is required for motor neurons in intersegmental nerve b to be guided to the correct muscles (Krueger et al., 1996). Also, LAR determines the size and strength of larval NMJs (Kaufmann et al., 2002). In the visual system, LAR is required for photoreceptor cells to defasciculate in order to target to the appropriate lamina (Clandinin et al., 2001), and LAR is required for correct targeting of the R7 photoreceptor neurons into the deep M6 layer of the medulla (Clandinin and Zipursky, 2002). Studying RPTPs further will help elucidate their 
mechanism of function, as well as help construct signaling pathways that mediate proper neural development.

CLR-1 is a RPTP with typical RPTP domains. There is one cysteine rich, one Immunoglobulin (Ig), and two Fibronectin type III (FnIII) extracellular domains. In addition, there is a transmembrane domain, and two intracellular phosphatase domains: one catalytic, one inactive. CLR-1 was initially studied for its role in inhibition of EGL15, the C. elegans homolog of the fibroblast growth factor receptor (FGFR), in the hypodermis (Kokel et al., 1998). Later, it was studied for its role in inhibition of the UNC-40/DCC axon guidance pathway in AVM touch-receptor neurons (Chang et al., 2004). AVM neurons are guided ventrally by the secreted UNC-6/Netrin ligand, which binds the UNC-40/DCC transmembrane receptor to mediate attraction to the ventral nerve cord, as well as by the secreted SLT-1/Slit ligand, which binds the SAX-3/Robo IgSF transmembrane receptor to mediate repulsion from the dorsal region. clr-1 single mutants do not have AVA axon guidance defects, but double-mutants with sax-3 or slt-1 have a dramatic increase in the penetrance of axon guidance defects over sax-3 or slt-1 single mutants, suggesting that CLR-1 negatively regulates UNC-40/DCC signaling during axon guidance (Chang et al., 2004). However, with regards to neural circuit formation, CLR-1 has only been studied previously for its role in axon guidance. In this study, we demonstrate a novel role for CLR-1 in SPR.

In this study, we find that CLR-1 functions with UNC-6/Netrin and UNC-40/DCC in SPR. However instead of antagonizing the function of UNC-40/DCC as it does in axon guidance, CLR-1 functions as a positive signal to UNC-40/DCC to mediate SPR. 
Additionally, we identify the defect in $c l r-1$ mutants as being specifically in SPR and not a secondary consequence of defects in polarity or protein targeting. We also find that many molecules that function with CLR-1 and other RPTPs do not have a conserved role in the CLR-1-mediated SPR pathway, likely indicating that this is a new signaling pathway. We hope that the characterization of this novel role for CLR-1 in SPR will give valuable insights into the molecular mechanisms of sensory neural circuit formation. 


\section{EXPERIMENTAL PROCEDURES}

\section{Strains and genetics}

Wild-type strains were C. elegans var. Bristol, strain N2. All strains (unless otherwise noted) contain the integrated ${ }_{p} n l p-1:: m$ Cherry $(10 \mathrm{ng} / \mu \mathrm{l}),{ }_{p} f l p-18:: m$ Cherry $(5$

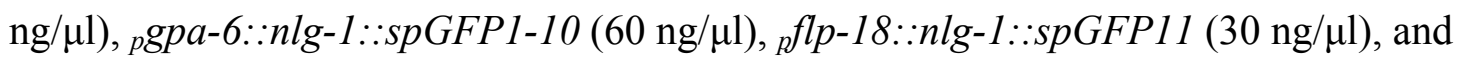
${ }_{p} O d r-1:: R F P(20 \mathrm{ng} / \mu \mathrm{l})$ transgene $w y I s 157 \mathrm{IV}$. All strains were raised on OP50 Escherichia coli-seeded NGM plates at $20^{\circ} \mathrm{C}$ and were maintained according to standard C. elegans methods (Brenner, 1974). Mutant strains used in this study include clr1(e1745ts) II, unc-40(e271) I, unc-6(ev400) X, bar-1(ga80) X, egl-15(n484) X, lin10(e1439) I, ina-1(gm39) III, and wyIs 157 IV (Park et al., 2011). The extrachromosomal transgenic array used for presynaptic and postsynaptic localization in wild type and $c l r-1$ was wyEx2309 in Figure 2A-D ( $n$ nlp-1::mCherry::rab-3 (1 ng/ $\mu 1),{ }_{p} f l p-18:: n l g-1:: Y F P$

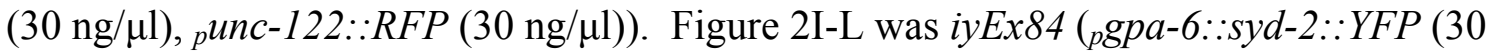
$\left.\mathrm{ng} / \mu \mathrm{l}),{ }_{p} u n c-122:: \operatorname{RFP}(20 \mathrm{ng} / \mu \mathrm{l})\right)$, in wild type and $c l r-1$. In wild type and $c l r-1$, Figure

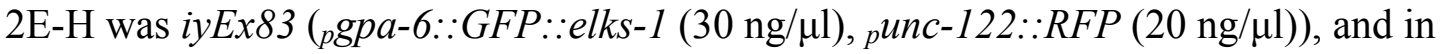
wild type, $c l r-1$ in Figure $2 \mathrm{M}-\mathrm{P}$ was $i y E x 82\left({ }_{p} f l p-18:: n l g-1:: Y F P(30 \mathrm{ng} / \mu \mathrm{l}),{ }_{p} n l p-\right.$

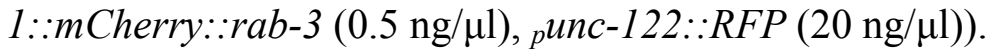

\section{Fluorescent microscopy}

Images were taken of live C. elegans worms using a Carl Zeiss Axio Imager.A1 fluorescent microscope at $630 \mathrm{X}$ magnification. All micrographs were taken at the larval 4 (L4) stage, except for the synaptic component experiments, for which they were taken at the larval 2 (L2) stage. To anesthetize worms, a 2:1 ratio of $0.3 \mathrm{M}$ 2,3-butanedione 
monoxime (BDM) to $10 \mathrm{mM}$ levamisole solution was utilized, which did not influence SPR phenotypes.

\section{Phenotypic quantification}

NIH ImageJ software (Abramoff, 2004) was used to quantify data from images. This data included PHB-AVA NLG-1 GRASP intensity, ${ }_{p} n l p-1:: m$ Cherry::rab-3 intensity, PHB-AVA neurite contact, and NLG-1 GRASP synaptic length. The remaining methods described were based on methods we previously used (Park et al., 2011). Intensity for NLG-1 GRASP, mCherry::RAB-3, GFP::ELKS-1, SYD-2::YFP, and NLG-1::YFP was determined by outlining each group of puncta and measuring the intensity at each pixel. To adjust for differences in background fluorescence, the background intensity was approximated by determining the minimum intensity value in a region adjacent to the puncta. This value was then subtracted from the intensity for each pixel. Then, the sum of the adjusted values was calculated. Median intensity values were normalized to wild-type levels measured on the same day and under the same microscope.

\section{Statistical analysis}

Median values for relative intensity were compared by a Wilcox u-test, which compares the medians of two independent groups. If more than one u-test was conducted for a group of data presented in the same experiment, the p-values were adjusted for multiple comparisons by the Hochberg method. The Hochberg method is a standard procedure used to adjust for the tendency to reject incorrectly a null hypothesis when multiple comparisons are made. The Hochberg method can only conservatively increase 
$\mathrm{p}$-values. Results are reported in the form of $\mathrm{p}$-values in the figures in this thesis $\left({ }^{*} \mathrm{p}<\right.$ $0.05, * * \mathrm{p}<0.01, * * * \mathrm{p}<0.001, \mathrm{NS} \mathrm{p}>0.05)$, and exact $\mathrm{p}$-values are given in Table 1 rather than error bars. Error bars are not utilized since intensity readings are not based on a normal distribution, which is required to employ error bars. 


\section{RESULTS}

\section{clr-1 mutants have defective synaptic partner recognition (SPR)}

For this study, we utilized Neuroligin-1 GFP-Reconstitution Across Synaptic

Partners (NLG-1 GRASP) to label synapses in vivo between the two PHB sensory

neurons and the two AVA interneurons. We also used cytosolic mCherry to label neurite contact as a means to assay SPR in mutant animals. Previously, these markers were integrated into the genome to create the marker strain wyIs 157 and this strain was used to discover a novel UNC-6/Netrin and UNC-40/DCC-mediated SPR pathway (Park et al., 2011). To identify genes that function in this process, we tested 35 different molecules that are directly or indirectly implicated in UNC-6/Netrin and UNC-40/DCC-mediated neuronal cell migration and/or axon guidance pathways. We crossed the strain wyIs 157 into these 35 candidate mutant strains. Several molecules, including the canonical UNC6/Netrin and UNC-40/DCC-mediated axon guidance and cell migration pathway members UNC-115/abLIM, UNC-5/UNC5, UNC-129/TGF $\beta$, AGE-1/PI3K, and CLEC38 displayed no abnormal SPR phenotypes (Park et al., 2011). However, clr-1, which encodes a transmembrane receptor protein tyrosine phosphatase (RPTP), had highly penetrant, very severe defects in SPR. In clr-1(e1745) II mutants, which contain a temperature-sensitive allele that deactivates the catalytic phosphatase domain, neurite contact was not defective, but NLG-1 GRASP signal was reduced to $15 \%$ of wild-type levels (Figure 1G, H, Q). This is a defect similar to that in unc-6/Netrin and unc-40/DCC mutants. In our previous studies, we found that NLG-1 GRASP intensity in unc-6/Netrin and $u n c-40 / D C C$ mutants was $45-55 \%$ of wild type, a highly significant difference. This 
indicates that like UNC-6/Netrin and UNC-40/DCC, CLR-1 promotes SPR pathway function. This contrasts with CLR-1's function in axon guidance, which is to antagonize UNC-6/Netrin and UNC-40/DCC function (Chang et al., 2004).

\section{CLR-1 functions in the UNC-6/Netrin and UNC-40/DCC-mediated SPR pathway}

Since $c l r-1$ mutants have a highly penetrant and very severe SPR defect, we wanted to determine whether CLR-1 functions in the UNC-6/Netrin and UNC-40/DCCmediated SPR pathway or in a parallel SPR pathway. We constructed double mutants between $c l r-1(e 1745) I I$ and $u n c-6(e v 400) X$ and $c l r-1(e 1745) I I$ and $u n c-40(e 271) I$. Both the unc- 6 and unc-40 mutations tested are caused by null alleles. If the defect in NLG-1 GRASP signal in the double mutants is not significantly different from the defect in $c l r-1$ mutants, then these genes likely function in the same pathway. However, if the defect in the double mutants is significantly more defective than in the $c l r-1$ single mutants, then the genes likely function in parallel pathways to mediate SPR. We found in both $c l r-1$; unc- 6 and in $c l r-1$; unc-40 double mutants that NLG-1 GRASP signal is not significantly different from that in $c l r-1$ single mutants, indicating that CLR-1 functions in the same pathway as UNC-6/Netrin and UNC-40/DCC to mediate SPR (Figure 1Q). To confirm our findings, we made animals that were heterozygous for each gene as well as trans-heterozygous for each gene combination. We produced and tested the heterozygotes, $c l r-1(e 1745) /+I I$, unc- $6(e v 400) /+X$, unc-40(e271)/+I, and the transheterozygotes, $\operatorname{clr}-1(e 1745) /+I I$; unc- $6(e v 400) /+X$, and $c l r-1(e 1745) /+I I$; unc40(e271)/+ I. Each single heterozygote should have had wild-type NLG-1 GRASP levels since each lesion is caused by a recessive allele, and indeed we found that each single 
gene heterozygote had wild-type NLG-1 GRASP intensity (Figure 1Q). A more severe defect in NLG-1 GRASP signal in trans-heterozygotes, as compared to wild-type animals, would indicate that CLR-1 functions in the same pathway as UNC-6/Netrin and UNC-40/DCC. This would likely be due to a reduction in gene product at two points in the pathway. Indeed, the trans-heterozygotes for $c l r-1$ and unc-6/Netrin and clr-1 and unc-40/DCC had severe defects in NLG-1 GRASP signal, again supporting a role for CLR-1 in the UNC-6/Netrin and UNC-40/DCC SPR pathway (Figure 1Q). Thus, CLR-1 likely functions in the same pathway as UNC-6/Netrin and UNC-40/DCC to mediate SPR. 

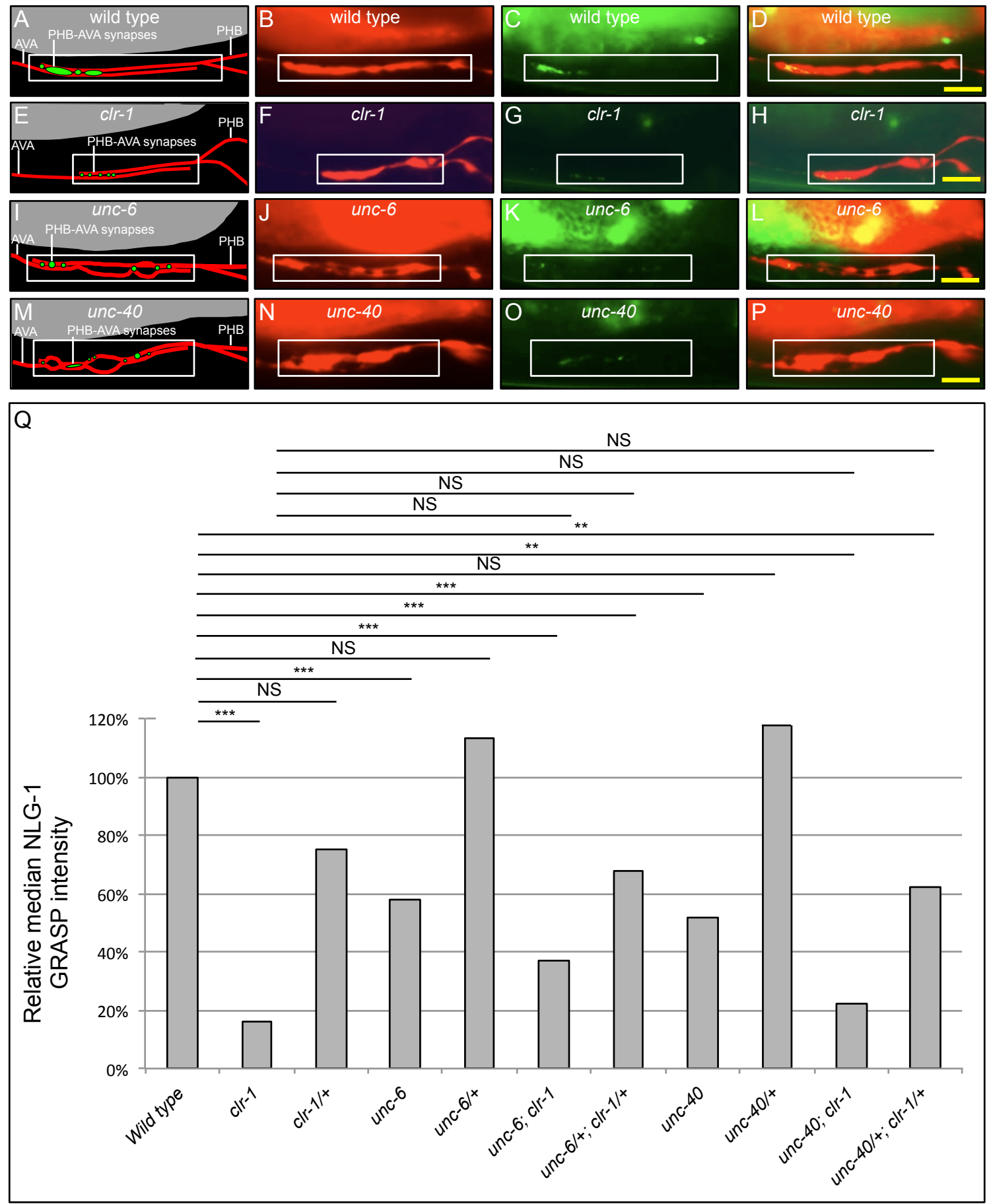
Figure 1. $c l r-1$ mutants display defective SPR and CLR-1 functions in the UNC6/Netrin and UNC-40/DCC-mediated SPR pathway.

(A) Schematic and (B, C, D) micrographs of a wild-type animal. (B) mCherry labels PHB and AVA neurite adhesion, and is specifically ${ }_{p} P H B:: m$ Cherry, ${ }_{p} A V A:: m$ Cherry.

(C) The NLG-1 GRASP signal indicates synapses between PHB and AVA neurons, and is specifically ${ }_{p} P H B n l g-1:: s p G F P 1-10,{ }_{p} A V A n l g-1: \because s p G F P 11$. (D) Merged image. (E) Schematic and (F, G, H) micrographs of a clr-1 mutant. (E, G) The NLG-1 GRASP signal is highly reduced. (K) Merge. (I) Schematic and (J, K, L) micrographs of an unc6 mutant animal. (I, K) The reduced NLG-1 GRASP signal is similar to that in unc-40 animals $(\mathrm{M}, \mathrm{O}) . \quad(\mathrm{J}, \mathrm{N})$ Defects in PHB and AVA neurite contact are also similar. (L, P) Merged image. (D, H, L, P) Yellow scale bar, $2 \mu \mathrm{m}$. (Q) Quantification of reduction in NLG-1 GRASP fluorescence in $c l r-1, u n c-6$ and $u n c-40$ mutants using NIH ImageJ. For wild type $\mathrm{n}=94, \mathrm{clr}-1 \mathrm{n}=80$, unc- $6 \mathrm{n}=87$, and $u n c-40 \mathrm{n}=85$ animals. ${ }^{* * *} \mathrm{p}<0.001$, u-test. For wild type and $c l r-1, \mathrm{p}=1.6^{\prime} 10^{-9}$, wild type and $u n c-6, \mathrm{p}=1.0^{\prime} 10^{-5}$, wild type and unc$40, \mathrm{p}=1.0^{\prime} 10^{-7}$. For $u n c-6$ and $u n c-40, \mathrm{p}=5.8^{\prime} 10^{-1}$. P-values were adjusted for multiple comparisons using the Hochberg method. Quantification of contact defects between PHB and AVA neurites using NIH ImageJ (data not shown in graph, but displayed in micrographs F, J, N, schematics E, I, M). The same animals for NLG-1 GRASP analysis were used. For $u n c-6$ and $u n c-40: * * * \mathrm{p}<0.001$, for $c l r-1$ : NS, $\mathrm{p}>0.05$, t-test. For wild type and $c l r-1, \mathrm{p}=9.0^{\prime} 10^{-1}$. For wild-type and $u n c-6, \mathrm{p}=2.4^{\prime} 10^{-1} 18$. For wild-type and unc-40, $\mathrm{p}=2.4^{\prime} 10^{-1} 18$. For double mutant analysis, wild type $\mathrm{n}=40, c l r-1 \mathrm{n}=80$, unc-6; clr$1 \mathrm{n}=20$, unc-40; clr-1 $\mathrm{n}=32$ animals. NS, $\mathrm{p}>0.05$, $* * * \mathrm{p}<0.001, * * \mathrm{p}<0.01$, u-test. For $c l r-$

1 and $u n c-6 ; c l r-1, \mathrm{p}=5.4^{\prime} 10^{-2}$. For wild type and unc-6; $c l r-1, \mathrm{p}=7.8^{\prime} 10^{-4}$. For $\operatorname{clr}-1$ and unc-40; clr-1, $\mathrm{p}=3.8^{\prime} 10^{-1}$. For wild type and unc-40; $c l r-1, \mathrm{p}=4.2^{\prime} 10^{-3}$. For heterozygote analysis, wild type $\mathrm{n}=40, \mathrm{clr}-1 /+\mathrm{n}=38$, unc-6/+ $\mathrm{n}=39$, unc $-40 /+\mathrm{n}=24$. NS, $\mathrm{p}>0.05$, utest. For wild type and $c l r-1 /+, p=1.7^{\prime} 10^{-1}$. For wild type and $u n c-6 /+, p=8.7^{\prime} 10^{-1}$. For wild type and $u n c-40 /+, \mathrm{p}=3.8^{\prime} 10^{-1}$. For trans-heterozygote analysis, wild type $\mathrm{n}=40$, unc-6/+; clr $-1 /+\mathrm{n}=38$, unc $-40 /+;$ clr $-1 /+\mathrm{n}=10$. $* * * \mathrm{p}<0.001, * * \mathrm{p}<0.001 \mathrm{u}$-test. For wild type and $u n c-6 /+; c l r-1 /+, \mathrm{p}=4.3^{\prime} 10^{-4}$. For wild type and $u n c-40 /+; c l r-1 /+$, $\mathrm{p}=4.0^{\prime} 10^{-3}$.

\section{Synaptic components are properly localized in $c l r-1$ mutants}

We propose that defects in synaptogenesis in clr-1 mutants could indicate a failure in SPR. Another possible explanation is that CLR-1 specifies localization of presynaptic components to the correct region of the PHB neurite. Such a role was observed for UNC-6/Netrin and UNC-40/DCC in AIY interneurons (Colón-Ramos et al., 2007) Thus, the failure to form PHB-to-AVA synapses in the $c l r-1$ mutants could be a secondary consequence of improper localization of synaptic components. However, 
UNC-6/Netrin and UNC-40/DCC had no such role in PHB (Park et al., 2011). If CLR-1 did play a role in localizing presynaptic components, we would predict a loss of presynaptic and/or postsynaptic components in the region of contact between the PHB and AVA neurites. To test this hypothesis, we assayed several well-characterized synaptic and vesicle markers in the $c l r-1$ mutant background. We crossed strains containing the presynaptic vesicle trafficking marker mCherry::RAB-3 expressed in PHB, the active zone markers GFP::ELKS-1 or SYD-2::YFP expressed in PHB, and the postsynaptic marker NLG-1::YFP expressed in AVA, into $c l r-1$ mutants (Park et al., 2011). Interestingly, we found that $c l r-1$ mutants do not have differences in any of these synaptic markers as compared to wild type (Figure 2Q). Specifically, we found that the presynaptic vesicle marker mCherry::RAB-3 and the presynaptic active zone markers SYD-2/Liprin- $\alpha:: Y F P$ and GFP::ELKS-1 are properly localized to the synaptogenic region of PHB in $c l r-1$ mutants. Similarly the postsynaptic marker NLG-1::YFP also was consistently localized to the region of AVA that forms synapses with PHB neurons. This is consistent with our previous findings for unc-6/Netrin and unc-40/DCC mutants (Park et al., 2011), and suggests that the SPR defect we observe in clr-1 mutants is a primary defect resulting from improper recognition of correct synaptic partners. 

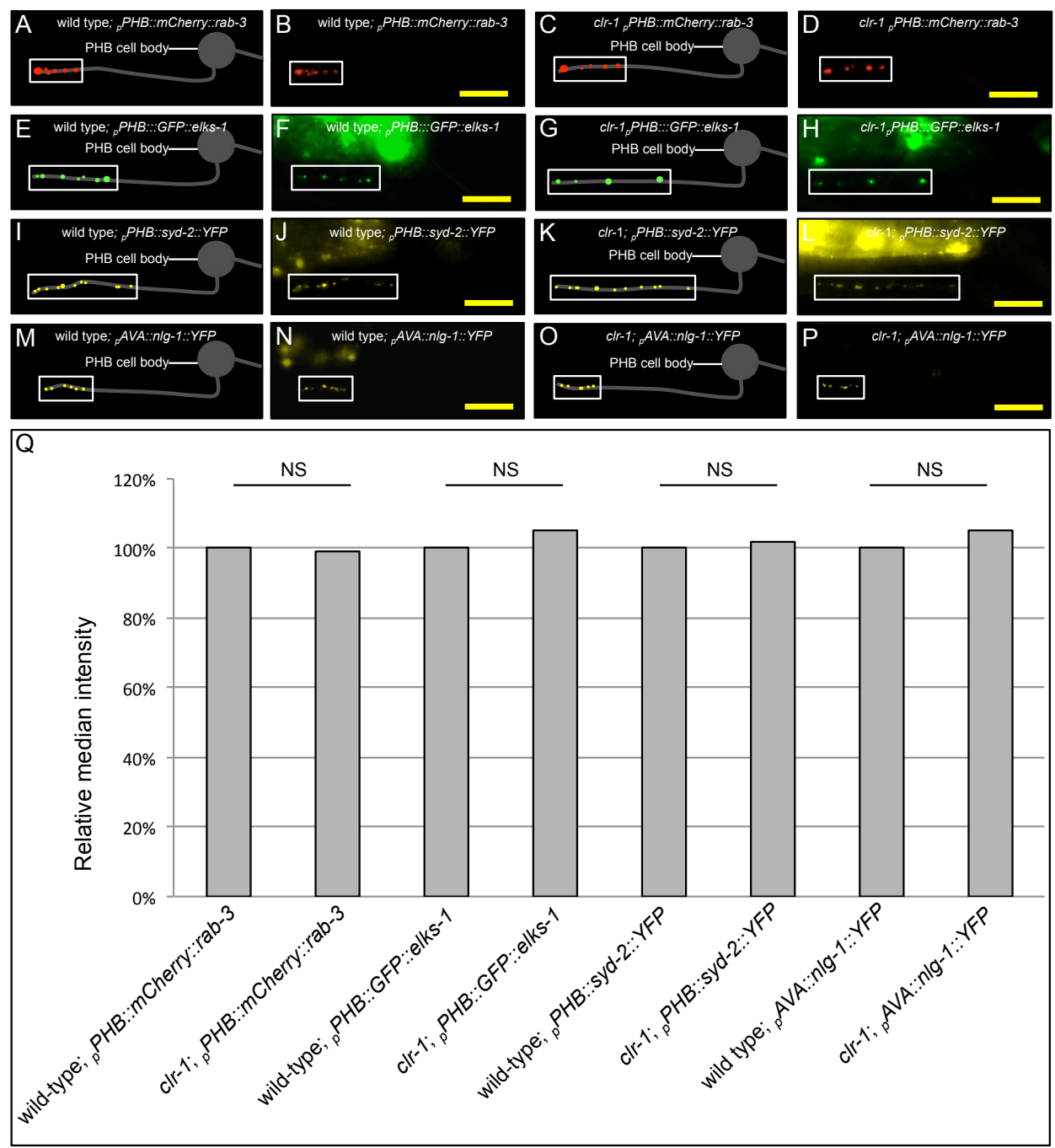

Figure 2. Vesicle trafficking, active zone, and postsynaptic components localize to the correct subcellular compartment in clr-1 mutants.

(A, B, E, F, I, J, M, N) Wild type, (C, D, G, H, K, L, O, P) clr-1 are labeled with the presynaptic vesicle trafficking marker mCherry::RAB-3 (A-D), active zone markers SYD-2::YFP (I-L) or GFP::ELKS-1 (E-H) expressed in PHB neurons or the postsynaptic marker NLG-1::YFP (M-P) expressed in AVA neurons. (B, D, F, H, J, L, N, P) Yellow scale bars: $2 \mu \mathrm{m}$. (A-D) Presynaptic vesicle trafficking components are unaltered in $c l r-1$ mutant animals, localizing to the distal region of the PHB axon, where synapses form (boxed in white). (E-L) Presynaptic active zone components (M-P) and postsynaptic specializations in the preanal ganglion are also unaltered in clr-1 mutant animals, localizing to the distal region of the PHB or AVA axon where synapses are normally form (boxed in white). (Q) Quantification of pre- or postsynaptic marker fluorescence intensity using NIH ImageJ indicates no significant difference among wild 
type and $c l r-1$ mutant animals. For mCherry::RAB-3, wild type $\mathrm{n}=40, c l r-1 \mathrm{n}=24$. For SYD-2::YFP, wild type $n=40, c l r-1 \mathrm{n}=40$. For GFP::ELKS-1, wild type $\mathrm{n}=40, c l r-1$ $\mathrm{n}=40$. For NLG-1::YFP, wild type $\mathrm{n}=40$, clr $-1 \mathrm{n}=41$. NS, not significant, $\mathrm{p}>0.05$, u-test. P-values were adjusted for multiple comparisons using the Hochberg method.

\section{Molecules that function with CLR-1 or with RPTPs in other pathways do not mediate SPR}

We hypothesized that molecules that act with RPTPs in other biological pathways may also act with CLR-1 in SPR. To answer this question, and to further elucidate the pathway(s) mediating SPR, we tested various molecules known to act with CLR-1 or other RPTPs in other developmental pathways. We tested EGL-15/FGFR because CLR1 inhibits EGL-15/FGFR in the hypodermis to regulate fluid balance (Kokel et al., 1998), and we tested three proteins known to function with RPTPs in other developmental pathways: BAR-1/ $\beta$-catenin, INA-1/ $\alpha$-integrin, and LIN-10/PDZ. None of these mutants displayed defects in SPR (Figure 3U). This suggests that EGL-15/FGFR, BAR-1/ $\beta$ catenin, INA-1/ $\alpha$-integrin, and LIN-10/PDZ do not function with CLR-1 in SPR. Interestingly, this suggests that we have identified a novel RPTP signaling pathway. 

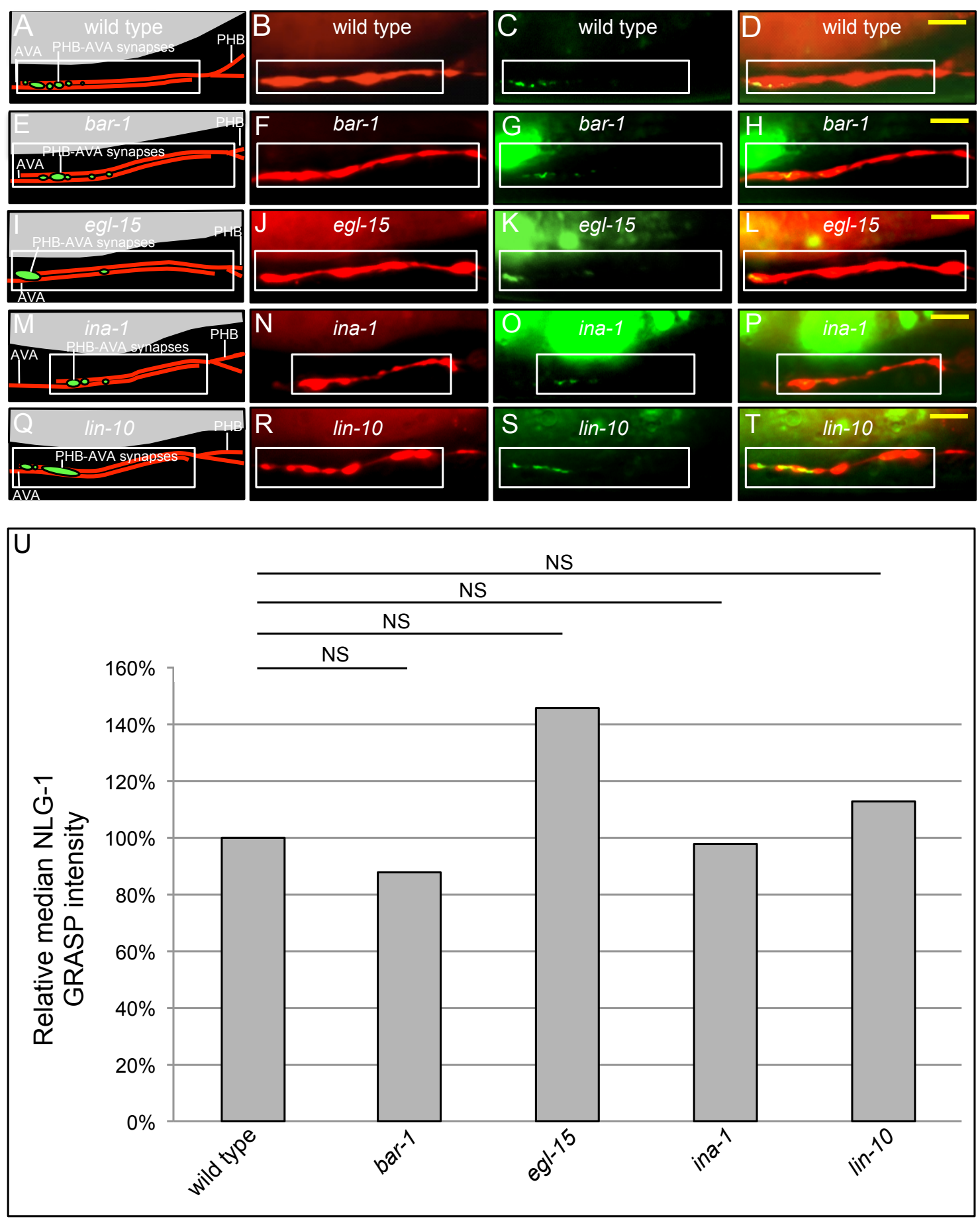
Figure 3. RPTP pathway mutants exhibit no defects in SPR.

Quantification (U) of NLG-1 GRASP intensity for bar-1, egl-15, ina-1, lin-10 mutants. Molecules that function with RPTPs, bar-1, egl-15, ina-1, and lin-10, have normal NLG1 GRASP intensities, indicating that SPR is molecularly distinct from previously studied RPTP signaling pathways. Wild-type $\mathrm{n}=40-50$, bar $-1 \mathrm{n}=35$, egl-15 $\mathrm{n}=80$, ina- $1 \mathrm{n}=$ 41 , lin- $10 \mathrm{n}=41$ animals. NS, not significant, u-test. P-values were adjusted for multiple comparisons using the Hochberg method. (A, E, I, M, Q) Schematics, (C, G, K, O, S) micrographs of normal PHB-AVA NLG-1 GRASP signal, (B, F, J, N, R) cytosolic mCherry labeling normal PHB and AVA neurite contact, and (D, H, L, P, T) merged images. (A-D) Wild type, (E-H) bar-1, (I-L) egl-15 (M-P) ina-1, (Q-T) and lin-10 animal. Yellow scale bar: $2 \mu \mathrm{m}$. 


\section{DISCUSSION}

Synaptic partner recognition (SPR) is a necessary step in neural circuit formation. Utilizing NLG-1 GRASP technology, we previously uncovered the role of the conserved UNC-6/Netrin and UNC-40/DCC ligand-receptor pair in SPR between PHB sensory neurons and AVA interneurons in C. elegans (Park et al., 2011). Using NLG-1 GRASP, our group has found that SPR is achieved through limiting amounts of UNC-6/Netrin secreted by postsynaptic AVA neurons, which interact in a juxtacrine manner with UNC40/DCC in the presynaptic PHB neurons. In this study, we discovered a novel role for the RPTP CLR-1 in promoting PHB-to-AVA SPR in the UNC-6/Netrin and UNC40/DCC pathway. Additionally, we revealed a novel mechanism for CLR-1, since it was previously found to antagonize UNC-6/Netrin and UNC-40/DCC-mediated pathways in earlier neural circuit formation steps. We also found that CLR-1 has a primary role in SPR and does not affect synaptic component localization. Finally, we discovered that molecules that function with RPTPs in other biological pathways are not conserved in the CLR-1-mediated SPR pathway, indicating that this is a new RPTP-signaling pathway.

\section{CLR-1 is required for SPR}

CLR-1 has been previously studied for regulating fluid balance in the hypodermis (Kokel et al., 1998), as well as in axon guidance in AVM touch-receptor neurons (Chang et al., 2004). Here, we suggest a novel role for CLR-1 in PHB sensory neuron to AVA interneuron SPR, a final step of neural circuit formation. The role of CLR-1 in SPR is particularly interesting because we previously found that PHB to AVA neurite contact in unc-6/Netrin and unc-40/DCC mutants was $80 \%$ of wild-type neurite contact, a highly 
significant decrease as compared to wild-type ( $\mathrm{p}<0.001)$ (Park et al., 2011). In contrast, clr-1 mutants do not have significant defects in neurite adhesion (Table 1). This suggests that although the PHB and AVA neurites are able to properly adhere, these neurons cannot recognize each other as correct partners with which to establish synaptic connections. 
Table 1. Summary of statistical analysis.

P-values for all statistical tests performed in this work.

\begin{tabular}{|c|c|c|c|c|}
\hline $\begin{array}{c}\text { Figure } \\
\text { Number }\end{array}$ & $\begin{array}{l}\text { Figure } \\
\text { Letter }\end{array}$ & Genotypes compared & $\begin{array}{c}\text { Statistical } \\
\text { Test }\end{array}$ & p-value \\
\hline 1 & $\mathrm{Q}$ & wild type and $c l r-1$ & u-test & $1.6 \mathrm{E}-09$ \\
\hline 1 & Q & wild type and $u n c-6$ & u-test & $1.0 \mathrm{E}-05$ \\
\hline 1 & Q & wild type and $u n c-40$ & u-test & $1.0 \mathrm{E}-07$ \\
\hline 1 & Q & wild type and unc-6; clr-1 & u-test & $7.8 \mathrm{E}-04$ \\
\hline 1 & Q & $u n c-6$ and $u n c-6 ; c l r-1$ & u-test & 2.6E-01 \\
\hline 1 & Q & $c l r-1$ and $u n c-6 ; c l r-1$ & u-test & $5.4 \mathrm{E}-02$ \\
\hline 1 & Q & wild type and $u n c-40 ; c l r-1$ & u-test & $4.2 \mathrm{E}-03$ \\
\hline 1 & Q & $u n c-40$ and $u n c-40 ; c l r-1$ & u-test & 4.4E-04 \\
\hline 1 & Q & $c l r-1$ and $u n c-40 ; c l r-1$ & u-test & $3.8 \mathrm{E}-01$ \\
\hline 1 & Q & wild type and $c l r-1 /+$ & u-test & $1.7 \mathrm{E}-01$ \\
\hline 1 & $\mathrm{Q}$ & wild type and unc-6/+ & $\mathrm{u}$-test & 8.7E-01 \\
\hline 1 & $\mathrm{Q}$ & wild type and $u n c-40 /+$ & u-test & $3.8 \mathrm{E}-01$ \\
\hline 1 & $\mathrm{Q}$ & wild type and unc- $6 /++$ clr- $1 /+$ & u-test & $4.3 \mathrm{E}-04$ \\
\hline 1 & Q & wild type and unc-40/+; clr-1/+ & u-test & $4.0 \mathrm{E}-03$ \\
\hline 1 & & wild type and $c l r-1$ & t-test & $9.0 \mathrm{E}-01$ \\
\hline 1 & & wild type and $u n c-6$ & t-test & $2.4 \mathrm{E}-18$ \\
\hline 1 & & wild type and $u n c-40$ & t-test & $2.4 \mathrm{E}-18$ \\
\hline 2 & $\mathrm{Q}$ & wild type; $m$ Cherry::rab-3, clr-1; mCherry::rab-3 & u-test & $9.1 \mathrm{E}-01$ \\
\hline 2 & Q & wild type; syd-2::YFP and $c l r-1 ;$ syd-2::YFP & u-test & $5.4 \mathrm{E}-01$ \\
\hline 2 & Q & wild type; GFP::elks-1 and clr-1; GFP::elks-1 & u-test & $5.0 \mathrm{E}-01$ \\
\hline 2 & Q & wild type; $n \lg -1: \because Y F P$ and $c l r-1 ; n \lg -1:: Y F P$ & u-test & $9.5 \mathrm{E}-01$ \\
\hline 3 & $\mathrm{U}$ & wild type and bar-1 & u-test & $5.9 \mathrm{E}-01$ \\
\hline 3 & $\mathrm{U}$ & wild type and egl-15 & u-test & $7.3 \mathrm{E}-02$ \\
\hline 3 & $\mathrm{U}$ & wild type and ina- 1 & u-test & $9.4 \mathrm{E}-01$ \\
\hline 3 & $\mathrm{U}$ & wild type and $\operatorname{lin}-10$ & u-test & 7.3E-01 \\
\hline
\end{tabular}

\section{CLR-1 functions in the UNC-6/Netrin and UNC-40/DCC-mediated SPR pathway}

Through double mutant and trans-heterozygote analysis, we found that CLR-1

likely functions in the same UNC-6/Netrin and UNC-40/DCC-mediated SPR pathway

that we previously identified (Park et al., 2011). We had hypothesized that CLR-1 would function in the UNC-6/Netrin and UNC-40/DCC pathway because of previous studies that placed CLR-1 as an upstream repressor of the receptor-ligand pair in axon guidance of touch-receptor neurons AVM (Chang et al., 2004). However, we found an opposite mechanistic role of CLR-1 in the SPR pathway, as CLR-1 promotes UNC-6/Netrin and 
UNC-40/DCC-mediated SPR. Not only did we find a novel role for CLR-1 in SPR, but we also found a novel mechanism by which CLR-1 functions.

A vital step in understanding the role of CLR-1 in the UNC-6/Netrin and UNC40/DCC-mediated SPR pathway is to determine the cell(s) in which CLR-1 functions. If CLR-1 functions in presynaptic PHB sensory neurons, it may, like UNC-40/DCC, bind UNC-6/Netrin secreted from postsynaptic AVA interneurons (Figure 4a). From our previous studies, we found that UNC-6/Netrin is secreted in limiting amounts from AVA (Park et al., 2011), which is also shown in this model. Alternately, if CLR-1 is expressed by postsynaptic AVA interneurons, CLR-1 could possibly bind secreted UNC-6/Netrin and present it across the synaptic cleft to UNC-40/DCC receptors expressed by presynaptic PHB sensory neurons, possibly recruiting postsynaptic components in AVA to completely transmit the signal (Figure $4 \mathrm{~b}$ ). This hypothesis is supported by previous studies on the Netrin-G ligand NGL-3 that forms trans-synaptic adhesions with the RPTP LAR in order to regulate excitatory synapses in vertebrates (Kwon et al., 2010). We would further define the role of CLR-1 by determining its subcellular localization. UNC6/Netrin is localized only to synaptic sites, whereas UNC-40/DCC is localized to the entire PHB axon length that contacts AVA (Park et al., 2011). Because UNC-6/Netrin is a limiting factor and assuming that CLR-1 functions similar to UNC-40/DCC, CLR-1 may also be localized to the region of neurite contact, binding UNC-6/Netrin only at synaptogenic sites. Co-localization experiments with UNC-6/Netrin and UNC-40/DCC could show if CLR-1 functions directly with these proteins at a specific site on the 
neurons. This will further characterize the role of CLR-1 in the UNC-6/Netrin and UNC40/DCC-mediated SPR pathway. 


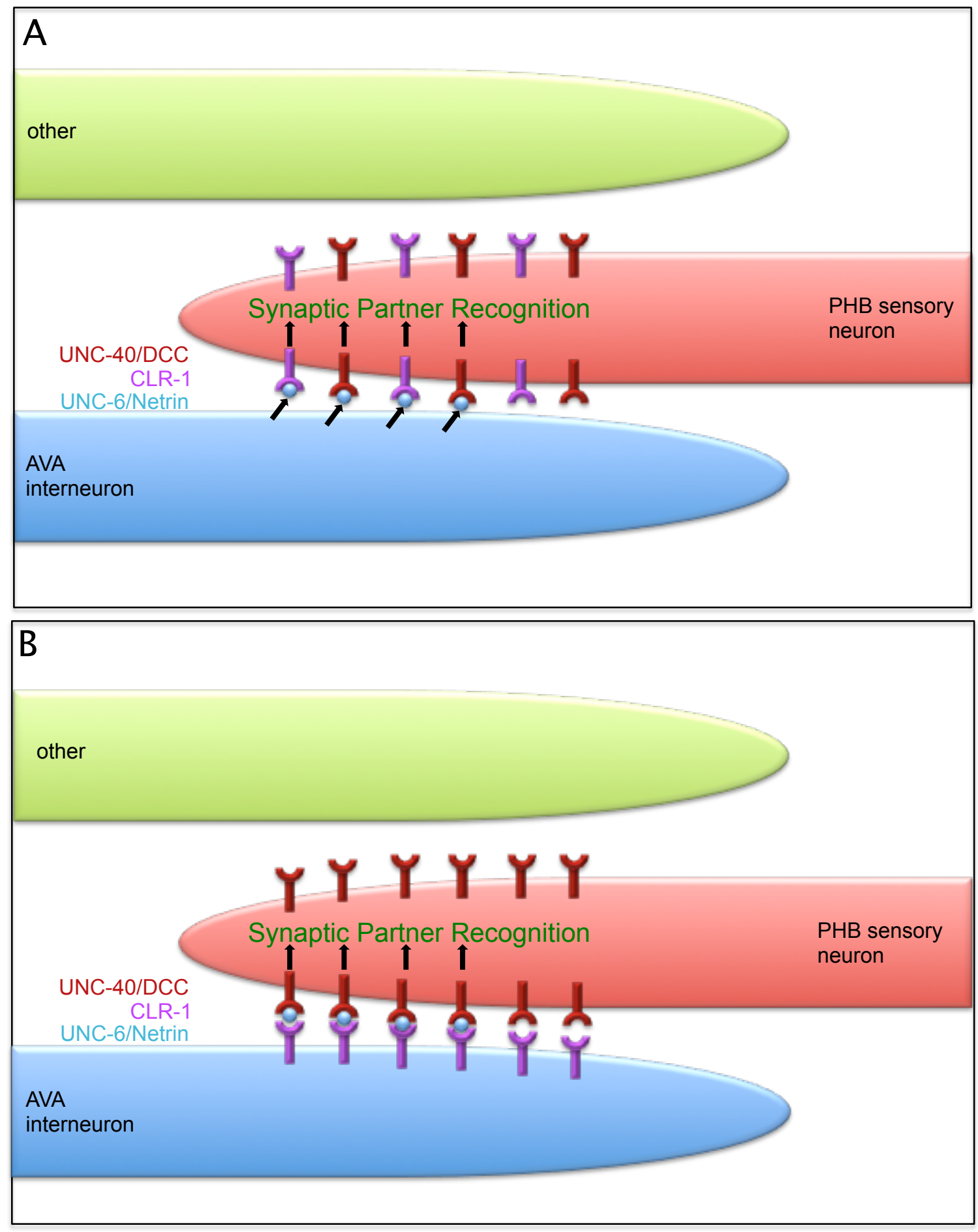


Figure 4. UNC-6/Netrin, UNC-40/DCC, and CLR-1 mediate SPR between presynaptic PHB sensory neurons and postsynaptic AVA interneurons.

In the first model (A), UNC-6 secreted from AVA interneurons binds either CLR-1 or UNC-40 expressed in PHB neurons to direct SPR. Limiting amounts of UNC-6 bind only a subset of CLR-1 or UNC-40 receptors in PHB, inducing a recognition event, resulting in synaptogenesis between the PHB and AVA neurons. In the second model (B), UNC-6 secreted from AVA interneurons binds CLR-1 also expressed by AVA interneurons, presenting UNC-6 across the synaptic cleft to UNC-40 expressed in PHB, inducing a recognition event between PHB and AVA neurons.

\section{Incorrect SPR in clr-1 mutants is the result of a primary recognition defect and not}

\section{due to incorrect synaptic component localization}

We found that in clr-1 mutants, vesicle trafficking, active zone, and postsynaptic fluorescent markers are properly localized to the synaptogenic regions at the distal end of each appropriate axon. This finding is similar to that found previously in unc-6/Netrin and $u n c-40 / D C C$ mutants (Park et al., 2011). Interestingly, this contrasts with a finding by Colón-Ramos and colleagues (2007), in which AIY interneurons express UNC40/DCC that binds UNC-6/Netrin secreted from non-neuronal, glia-like sheath cells to mediate the transport of presynaptic components to the neurite. Our work indicates that the SPR pathway may occur downstream of the synaptic component localization pathway. These findings were remarkable because they confirmed that the decrease in NLG-1 GRASP signal in the clr-1 mutants was not due to defects in protein trafficking or neuronal polarity. Instead, the defect is likely to result from a failure to identify the neurons with which to form synapses. 


\section{The CLR-1 SPR pathway is distinct from previously described RPTP pathways}

To fully characterize the CLR-1 mediated SPR pathway, we would need to discover additional molecules that function in the pathway. We attempted to do this by testing several candidates that have been found to act with RPTPs in other biological pathways, including EGL-15/FGFR, which is inhibited by CLR-1 in regulating fluid balance (Kokel et al., 1998), as well as BAR-1/ $\beta$-catenin, INA-1/ $\alpha$-integrin (Natarajan et al., 2001), and LIN-10/PDZ (Kawachi et al., 1999). Interestingly, we found that none of these proteins are conserved in the CLR-1-mediated SPR pathway (Figure 4Q). This result is exciting because this RPTP-signaling pathway is a novel one, previously unidentified. Also, this is the first evidence that CLR-1 can function independent of EGL-15/FGFR. In order to further elucidate the SPR pathway, a forward genetic suppressor screen of CLR-1 may prove fruitful in discovering new molecules that function with CLR-1 in mediating SPR.

\section{Implications of SPR in understanding proper brain function}

SPR is one of the final steps in neural circuit formation. First, cell bodies migrate to their proper position. Second, axons projected from neurons are guided by proteins called growth cues, which are normally secreted from non-neuronal guidepost cells. Third, synaptic components are properly localized to synaptogenic regions on the neurons. Next, a recognition event between correct synaptic partners occurs (SPR). Last, synapses are formed between correct neuronal partners. Very little work has been performed on the molecular mechanisms that mediate SPR. Identifying the molecules that mediate correct SPR will help elucidate this intricate process by which neurons 
correctly select other neurons with whom to form synapses. We have uncovered novel roles of UNC-6/Netrin, UNC-40/DCC, and CLR-1 in mediating SPR. Correct neural circuitry is vital because neural circuits are responsible for everything we do, think, and perceive. Incorrect recognition events between neurons will cause faulty wiring of these circuits and could have disastrous results. Previous research has implicated improper neural circuit formation, due to defects in SPR or synaptogenesis, in autism spectrum disorders or ASDs (Durand et al., 2007; Garber, 2007). Understanding which molecules are responsible for correct SPR and the mechanism by which they function could uncover what proteins to target with drugs and therapies in patients that have disorders caused by defects in these processes, as well as learn how the brain and possibly the entire nervous system function normally. 


\section{REFERENCES}

Abramoff, M.D., Magelhaes, P.J., and Ram, S.J. (2004). Image Processing with ImageJ. Biophotonics International 11, 36-42.

Brennan, P.A., and Keverne, E.B. (1997). Neural mechanisms of mammalian olfactory learning. Prog Neurobiol 51, 457-481.

Brenner, S. (1974). The genetics of Caenorhabditis elegans. Genetics 77, 71-94.

Chang, C., Yu, T.W., Bargmann, C.I., and Tessier-Lavigne, M. (2004) Inhibition of netrin-mediated axon attraction by a receptor protein tyrosine phosphatase. Science 305, 103-106.

Clandinin, T.R., Lee, C.H., Herman, T., Lee, R.C., Yang, A.Y., Ovasapyan, S., and Zipursky, S. L. (2001) Drosophila LAR regulates R1-R6 and R7 target specificity in the visual system. Neuron 32, 237-248.

Clandinin, T.R., and Zipursky, S.L. (2002) Making connections in the fly visual system. Neuron 35, 827-841.

Colón-Ramos, D.A., Margeta, M.A., and Shen, K. (2007). Glia promote local synaptogenesis through UNC-6 (Netrin) signaling in C. elegans. Science 318, 103-106.

Dickson, B.J. (2002). Molecular mechanisms of axon guidance. Science 298, 1959-1964.

Durand, C.M., Betancur, C., Boeckers, T.M., Bockmann, J., Chaste, P., Fauchereau, F., Nygren, G., Rastam, M., Gillberg, I.C., Anckarsater, H., Sponheim, E., Goubran-Boutros, H., Delorme, R., Chabane, N., Mouren-Simioni, M.C., de Mas, P., Bieth, E., Rogé, B., Héron, D., Burglen, L., Gillberg, C., Leboyer, M., and Bourgeron, T. (2007). Mutations in the gene encoding the synaptic scaffolding protein SHANK3 are associated with autism spectrum disorders. Nat Genet, 39, 25-27.

Feinberg, E.H., VanHoven, M.K., Bendesky, A., Wang, G., Fetter, R.D., Shen, K., and Bargmann, C.I. (2008). GFP Reconstitution Across Synaptic Partners (GRASP) defines cell contacts and synapses in living nervous systems. Neuron 57, 353-363.

Fox, A.N., and Zinn, K. (2005). The heparan sulfate proteoglycan syndecan is an in vivo ligand for the Drosophila LAR receptor tyrosine phosphatase. Curr Biol 15, 1701-1711.

Garber, K. (2007). Neuroscience. Autism's cause may reside in abnormalities at the synapse. Science, 317, 190-191. 
Gitai, Z., Yu, T.W., Lundquist, E.A., Tessier-Lavigne, M., and Bargmann, C.I. (2003). The netrin receptor UNC-40/DCC stimulates axon attraction and outgrowth through enabled and, in parallel, Rac and UNC-115/AbLIM. Neuron, 37, 53-65.

Goodman, C.S. (1996). Mechanisms and molecules that control growth cone guidance. Annu Rev Neurosci 19, 341-377.

Hall, D.H., and Russell, R.L. (1991). The posterior nervous system of the nematode Caenorhabditis elegans: serial reconstruction of identified neurons and complete pattern of synaptic interactions. J Neurosci 11, 1-22.

Hedgecock, E.M., Culotti, J.G., and Hall, D.H. (1990). The unc-5, unc-6, and unc-40 genes guide circumferential migrations of pioneer axons and mesodermal cells on the epidermis in C. elegans. Neuron, 4, 61-85.

Johnson, K.G., Tenney, A.P., Ghose, A., Duckworth, A.M., Higashi, M.E., Parfitt, K., Marcu, O., Heslip, T.R., Marsh, J.L., Schwarz, T.L., Flanagan, J.G., and Van Vactor, D. (2006). The HSPGs Syndecan and Dallylike bind the receptor phosphatase LAR and exert distinct effects on synaptic development. Neuron 49, 517-531.

Johnson, K.G., and Van Vactor, D. (2003). Receptor protein tyrosine phosphatases in nervous system development. Physiol Rev 83, 1-24.

Kaufmann, N., DeProto, J., Ranjan, R., Wan, H., and Van Vactor, D. (2002). Drosophila liprin-alpha and the receptor phosphatase Dlar control synapse morphogenesis. Neuron 34, 27-38.

Kawachi, H., Tamura, H., Watakabe, I., Shintani, T., Maeda, N., and Noda, M. (1999). Protein tyrosine phosphatase zeta/RPTPbeta interacts with PSD-95/SAP90 family. Brain Res Mol Brain Res 72, 47-54.

Kokel, M., Borland, C. Z., DeLong, L., Horvitz, H. R. and Stern, M. J. clr-1 encodes a receptor tyrosine phosphatase that negatively regulates an FGF receptor signaling pathway in Caenorhabditis elegans. Genes Dev 12, 1425-1437 (1998).

Krueger, N.X., Van Vactor, D., Wan H.I., Gelbart, W.M., Goodman, C.S., and Saito, H. (1996). The transmembrane tyrosine phosphatase DLAR controls motor axon guidance in Drosophila. Cell 84, 611-622.

Kwon, S.K., Woo, J., Kim, S.Y., Kim, H., and Kim, E. (2010). Trans-synaptic adhesions between netrin-G ligand-3 (NGL-3) and receptor tyrosine phosphatases LAR, proteintyrosine phosphatase delta (PTPdelta), and PTPsigma via specific domains regulate excitatory synapse formation. J Biol Chem 285, 13966-13978. 
Levy-Strumpf, N., and Culotti, J.G. (2007). VAB-8, UNC-73 and MIG-2 regulate axon polarity and cell migration functions of UNC-40 in C. elegans. Nat Neurosci, 10, 161168.

Li, X., Meriane, M., Triki, I., Shekarabi, M., Kennedy, T.E., and Larose, L. (2002). The adaptor protein Nck-1 couples the netrin-1 receptor DCC (deleted in colorectal cancer) to the activation of the small GTPase Rac1 through an atypical mechanism. J Biol Chem, 277, 37788-37797.

Lundquist, E.A., Herman, R.K., Shaw, J.E., and Bargmann, C.I. (1998). UNC-115, a conserved protein with predicted LIM and actin-binding domains, mediates axon guidance in C. elegans. Neuron, 21, 385-392.

Moore, S.W., Tessier-Lavigne, M., and Kennedy, T.E. (2007). Netrins and their receptors. Adv Exp Med Biol 621, 17-31.

Natarajan, L., Witwer, N.E., and Eisenmann, D.M. (2001). The divergent Caenorhabditis elegans beta-catenin proteins BAR-1, WRM-1 and HMP-2 make distinct protein interactions but retain functional redundancy in vivo. Genetics 159, 159-172.

Park, J., Knezevich, P., Wung, W., O'Hanlon, S.N., Goyal, A., Benedetti, K.L., BarsiRhyne, B.J., Raman, M., Mock, N., Bremer, M., and VanHoven, M.K. (2011). A conserved juxtacrine signal regulates synaptic partner recognition in C. elegans. Neural Dev 6, 28

Serafini, T., Kennedy, T.E., Galko, M.J., Mirzayan, C., Jessell, T.M., and TessierLavigne, M. (1994). The netrins define a family of axon outgrowth-promoting proteins homologous to C. elegans UNC-6. Cell 78, 409-424.

Shen, K., Fetter, R.D., and Bargmann, C.I. (2004) Synaptic specificity is generated by the synaptic guidepost protein SYG-2 and its receptor, SYG-1. Cell 116, 869-81.

Shen, K., and Scheiffele, P. (2010). Genetics and Cell Biology of Building Specific Synapse Connectivity. Annu Rev Neurosci 33, 473-507.

Tessier-Lavigne, M., and Goodman, C.S. (1996). The molecular biology of axon guidance. Science 274, 1123-1133.

White, J.G., Southgate, E., Thomson, J.N., and Brenner, S. (1976). The structure of the ventral nerve cord of Caenorhabditis elegans. Philos Trans R Soc Lond B Biol Sci 275, $327-348$. 
White, J.G., Southgate, E., Thomson, J.N., and Brenner, S. (1986). The structure of the nervous system of the nematode $C$. elegans. Philos Trans R Soc Lond B Biol Sci 314, 1 340 .

Zhen, M., and Jin, Y. (1999) The liprin protein SYD-2 regulates the differentiation of presynaptic termini in C. elegans. Nature 401,371-375. 